ORIGINAL ARTICLE

\title{
Coxsackievirus B3 sequences in the myocardium of fatal cases in a cluster of acute myocarditis in Greece
}

\author{
N Spanakis, E N Manolis, A Tsakris, S Tsiodras, T Panagiotopoulos, G Saroglou, N J Legakis
}

J Clin Pathol 2005;58:357-360. doi: 10.1136/icp.2004.020099

See end of article for authors' affiliations

.....................

Correspondence to:

Dr A Tsakris, Department

of Microbiology, Medical

School, University of

Athens, 75 Mikras Asias

Street, 11527 Athens

Greece; atsakris@med.

voa.gr

Accepted for publication 8 July 2004

\begin{abstract}
Aim: The investigation of three fatal cases during a nationwide cluster of cases of an upper respiratory tract infection (URTI) associated with myocarditis and/or pericarditis in Greece in 2002.

Methods: In the three women who died, necropsies were performed and tissue sections were taken for histological examination, antigen detection by immunohistochemistry and indirect immunofluorescence assay (IFA), amplification of viral genomes by nested reverse transcription polymerase chain reaction (RT$P(R)$, and sequence analysis.

Results: All samples showed histological signs of active myocarditis. Immunohistochemistry revealed the presence of the enterovirus VP1 family of proteins and IFA revealed the presence of coxsackievirus B3 antigen. Nested RT-PCR amplified enteroviral alleles of the 5'-untranslated region which were identical to each other and to the coxsackievirus B3 sequences.

Conclusions: This study provides pathological evidence of enteroviral infection among fatal myocarditis cases in a nationwide URTI cluster of cases associated with myocarditis and/or pericarditis.
\end{abstract}

$\mathrm{V}$ ral infections of the heart are important causes of morbidity and mortality in all ages. Enteroviruses, and especially the coxsackievirus B family, are thought to be the most common cause of viral myocarditis, and may be detected in more than $25 \%$ of sporadic cases of acute onset or dilated cardiomyopathy. ${ }^{1-3}$ However, reports showing direct evidence of enterovirus induced acute upper respiratory tract syndrome associated with fatal cases of myocarditis and/or pericarditis are limited.

From January to April 2002, a nationwide investigation of a cluster of cases of acute upper respiratory tract syndrome associated with myocarditis and/or pericarditis took place in Greece. ${ }^{4}$ Three fatal cases as a result of acute myocarditis initiated the epidemiological investigation. Fifty one cases of myocarditis and/or pericarditis were reported from almost all regions of the country during March and April 2002.5 They were defined as clinically and laboratory confirmed cases of myocarditis and/or pericarditis that developed within 10 days of onset of viral infection symptoms that included signs of upper respiratory tract infection (URTI), along with pleyrodynia or myalgia. In a few of these cases, an enteroviral strain was indicated as the aetiological agent by virological or polymerase chain reaction (PCR) tests conducted on specimens from non-sterile sites, such as throat swabs or stool specimens (N Spanakis et al, unpublished data, 2002).

"Reports showing direct evidence of enterovirus induced
acute upper respiratory tract syndrome associated with
fatal cases of myocarditis and/or pericarditis are limited"

Although these results indicate a precedent enteroviral infection as the cause of these cases, they do not implicate enteroviruses as the causative agents for the three fatal myocarditis cases that occurred during this time period. The fatal cases occurred in the island of Crete, which has a maximum length of $260 \mathrm{~km}$ and a geographical surface of $8336 \mathrm{~km}^{2}$, in two cities that are $70 \mathrm{~km}$ apart (two cases in Heraklion and one case in Sitia). In our current study, the histological and molecular studies of the three fatal cases are presented.

\section{METHODS}

The first fatal case involved a 50 year old woman and was reported in January 2002, two months before the initiation of the investigation. The woman deteriorated rapidly and died 12 hours after admission to hospital. She had symptoms of URTI, myalgia, and chest pain during the last four days. The other two cases occurred during the time period of the investigation and fulfilled the case definition criteria. The second case occurred on April 2002 and concerned a 48 year old woman. Five days before her admission, she developed fever $\left(38.5^{\circ} \mathrm{C}\right)$, myalgia, chest pain, and a non-productive cough. Three days later, the fever subsided but her chest pain worsened and she was admitted to hospital. Laboratory tests disclosed high concentrations of creatine phosphokinase-MB and lactate dehydrogenase. Low potentials were seen in the electrocardiogram, and an ultrasound examination disclosed an ejection fraction of $15 \%$ and a moderate amount of pericardial fluid. During hospitalisation, she developed tachycardia and low blood pressure, which evolved to shock and resulted in her death. The third fatal case (April 2002) also involved a 48 year old woman. She presented to the outpatient department with fever $\left(38.7^{\circ} \mathrm{C}\right)$, myalgia, arthralgia, and malaise. During the first hours of her admission she developed low blood pressure, tachycardia, chest pain, and dyspnoea. An electrocardiogram showed ST elevation and an ultrasound examination showed a large amount of pericardial fluid. During the course of her disease she developed congestive heart failure and cardiogenic shock, which caused her death.

Necropsies of the three women who died were performed during the first 12 hours after death. Tissue sections were taken from 10 distinct myocardial sites from each patient and fixed in $10 \%$ buffered formalin and embedded in paraffin wax. Histological examination was performed after haematoxylin and eosin staining. Enteroviral antigen detection was performed by immunohistochemistry using a mouse

Abbreviations: IFA, indirect immunofluorescence assay; PCR, polymerase chain reaction; RT, reverse transcriptase; URTI, upper respiratory tract infection; 5'-UTR, 5'-untranslated region; VP1, viral protein 1 


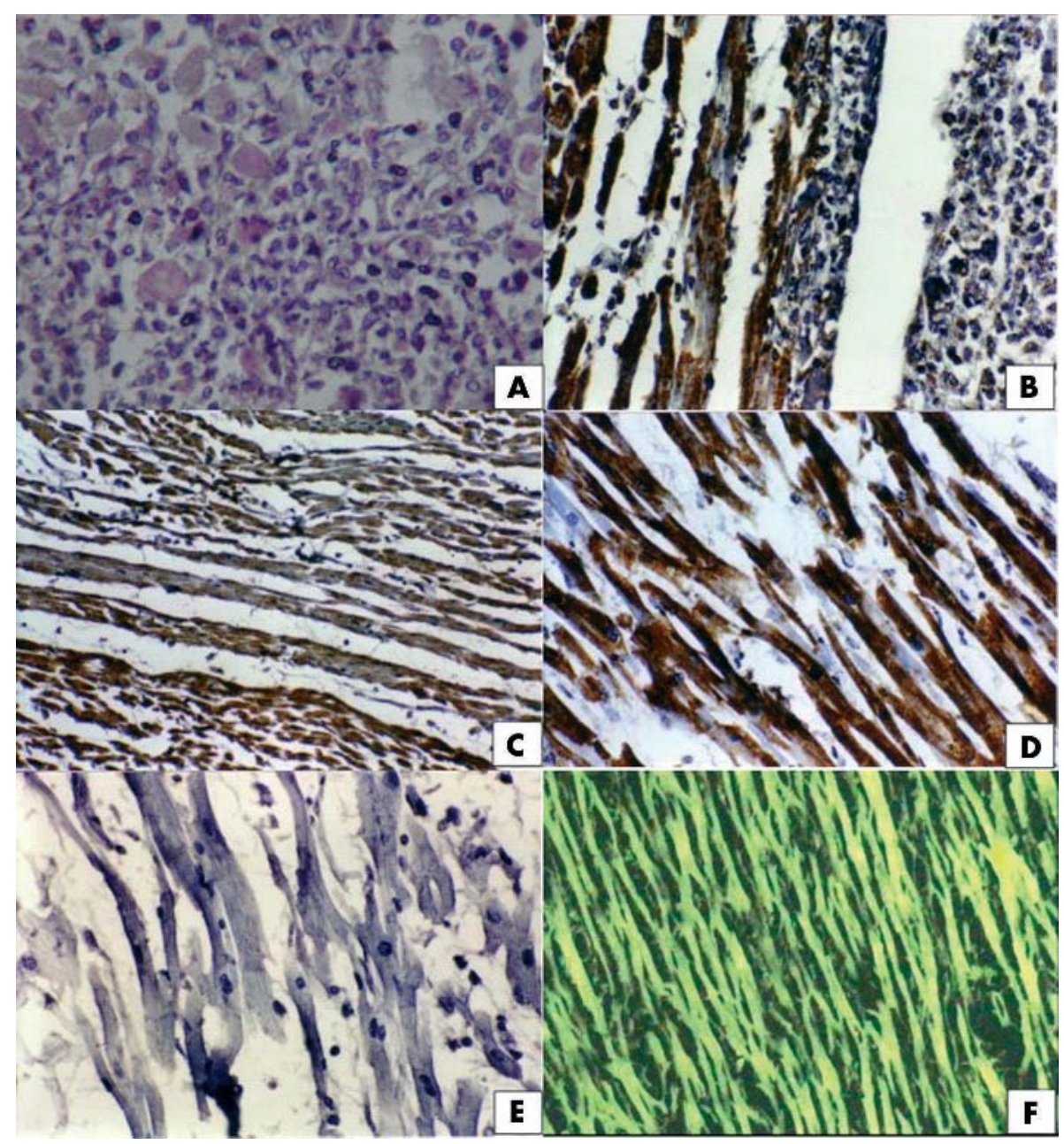

Figure 1 (A) Haematoxylin and eosin staining of myocardium from the first case showing interstitial infiltration consisting primarily of macrophages and lymphocytes (original magnification, $\times 100$ ). (B) Immunohistochemical staining of cardiac muscle cells for enterovirus showing strong cyłoplasmic reactivity (brown) in a tissue section from the first case. Note the interstitial infiltration by macrophages and lymphocytes on the right, together with pronounced damage to the muscle cells (original magnification, $\times 100$ ). (C) Immunohistochemical staining of cardiac muscle cells for enterovirus showing strong cytoplasmic reactivity in a tissue section from the second case (original magnification, $\times 50$ ). (D) Immunohistochemical staining of cardiac muscle cells for enterovirus in a tissue section from the third case. Note the damage to cells as a result of viral replication and/or inflammation (original magnification, $\times 100$ ). (E) Absence of immunohistochemical staining of cardiac muscle cells for adenovirus in a tissue section from the third case (original magnification, $\times 100$ ). (F) Detection of coxsackievirus B3 antigen by indirect immunofluorescence assay in cardiac muscle cells in a tissue section from the third case (original magnification, $\times 100$ ).

monoclonal antibody (clone 5-D8/1; Dako A/S, Glostrup, Denmark) that reacted with an epitope in the $\mathrm{N}$-terminus of capsid protein VPl, which is highly conserved in the enterovirus genus. Adenoviral antigen testing was done using a mouse monoclonal cocktail (Ab-5 cocktail; NeoMarkers, Freemont, California, USA) that reacted with the adenovirus early gene product of the ElA region. A biotinylated rabbit antimouse secondary antibody was used together with an avidin-biotinylated horseradish peroxidase complex (ABC staining system; Santa Cruz Biotechnology, Santa Cruz, California, USA). Specific detection of coxsackievirus B3 antigen was performed by indirect immunofluorescence assay (IFA) using the mouse anti-coxsackievirus B3 monoclonal antibody MAB948 according to the instructions of the manufacturer (Chemicon International, Temecula, California, USA).

Fresh tissue samples from the same origin were frozen at $-70^{\circ} \mathrm{C}$ for subsequent molecular studies. Extraction of total DNA from the tissue samples was performed using the Wizard Genomic Purification kit (Promega, Madison, Wisconsin, USA). Different PCR based methods were used for the detection of different viral types. Nested PCR was used for the detection of adenoviral DNA using generic primers that amplify a sequence inside the hexon protein coding region of the adenoviral genome. ${ }^{6}$ This method can detect the DNA of all 47 human adenovirus types in a wide range of clinical samples. A multiplex nested PCR method was applied for the detection and typing of human herpes viruses, specifically cytomegalovirus, Epstein-Barr virus, and herpes simplex virus. ${ }^{7}$ A first amplification step was performed using a mixture of non-degenerate oligonucleotides, capable of producing a mixture of fragments specific for each herpes virus type. These fragments were the substrate for a second multiplex reaction for which primers were designed to produce different sized amplicons for each related virus.

Total RNA extraction from the tissues was performed using the ToTALLY RNA kit (Ambion Inc, Austin, Texas, USA). The presence of the haemagglutination $\mathrm{A}$ and $\mathrm{B}$ genes was detected by a nested reverse transcription PCR (RT-PCR) method, as proposed by Herrmann et al. ${ }^{8}$ This method was used for a better specificity and sensitivity in detecting influenza A and B sequences. Enteroviral RNA was detected 
using a nested RT-PCR method that amplifies an enteroviral $5^{\prime}$-untranslated region (5'-UTR), as proposed by Read et al. ${ }^{9}$ The first step of the nested RT-PCR, which included the reverse transcription and first amplification, was performed using the Reverse-iT One-Step RT-PCR kit (Abgene, Epsom, Surrey, UK). The second amplification was performed immediately after using $2 \mu \mathrm{l}$ of the cDNA product, together with Thermoprime Plus DNA polymerase and a dNTP set (Abgene). Positive and negative DNA and RNA controls were included in all PCR experiments and genetic material was extracted twice from each sample. The expected $147 \mathrm{bp}$ amplicons of the $5^{\prime}$-UTR enteroviral region were purified by a Qiaex gel extraction kit (Qiagen, Chatsworth, California, USA), and were used as templates for sequencing of both strands by the ABI PRISM 377 DNA sequence analyser (Perkin Elmer, Applied Biosystems Division, Foster City, California, USA).

\section{RESULTS}

The postmortem study revealed alterations in cross morphology of the left and right ventricular wall, the intermedial septum, and the atrial cavity. In two of the three women (second and third cases), serous fluid was detected in the pericardial cavity. Macroscopic findings of dilated cardiomyopathy were not detected at postmortem examination. Apart from oedema of the lung, all other organs were histologically normal in all three patients.

According to the Dallas criteria, ${ }^{10}$ all three fatal cases fulfil the requirements for active myocarditis, characterised by diffuse inflammatory cell infiltration with concomitant necrosis. Signs of fibrosis and evidence of ischaemia were not shown. A pronounced interstitial and perivascular infiltration was present in the tissue sample from the fist patient, which consisted primarily of cells with the cytological features of macrophages and lymphocytes (fig lA), whereas interstitial infiltration was minimal in the second and third tissue samples. All tissues from the three cases examined by immunohistochemistry showed positive results for the presence of the enterovirus VPl family of proteins. Strong cytoplasmic reactivity was apparent in myocardial cells of the three different myocardial tissues (fig 1B-D). Adenoviral antigen was not detected in the samples (fig lE). When tissue samples were tested by IFA for the presence of coxsackievirus B3 antigen, strong reactivity was apparent in all three cases (fig $1 \mathrm{~F}$ ).

Viral DNA genes failed to amplify in all cases. Nested RTPCR was negative for influenza A and B but amplified enteroviral alleles in two separate sections of all three samples. BLAST nucleotide alignment showed that in all three cases the $147 \mathrm{bp} 5^{\prime}$-UTR amplimers were identical to each other, and were also identical to the coxsackievirus B3 sequences available on the database.

\section{DISCUSSION}

Cases of myocarditis and/or pericarditis may be a serious complication of several viral infections, and enteroviruses are often implicated as the causative agent in sporadic ${ }^{1-3}$ or epidemic cases. ${ }^{11}$ Enteroviral genomic sequences have also been detected in myocardium from cases of subacute or chronic endemic cardiomyopathy. ${ }^{12}$ In addition, enteroviral infections have been associated with rejection in heart transplant recipients, ${ }^{13}$ arrythmogenic right ventricular dysplasia/cardiomyopathy, ${ }^{14}$ cases of dilated cardiomyopathy, ${ }^{15}$ and ventricular arrythmias in survivors of sudden cardiac death. ${ }^{16}$ In a recent report, molecular techniques demonstrated the presence of enterovirus sequences in the heart tissue samples of 14 of 60 infants with the sudden infant death syndrome and in none of the 36 control samples. ${ }^{17}$ Together with a previous similar report, ${ }^{18}$ these publications

\section{Take home messages}

- We investigated three fatal cases during a nationwide cluster of cases of upper respiratory tract infection (URTI) associated with myocarditis and/or pericarditis and found that all samples showed histological signs of active myocarditis

- Immunohistochemistry revealed the presence of the enterovirus VP1 family of proteins and the indirect immunofluorescence assay revealed the presence of coxsackievirus B3 antigen

- Nested reverse transcription polymerase chain reaction amplified enteroviral alleles of the $5^{\prime}$-untranslated region that were identical to each other in the three cases and also identical to coxsackievirus B3 sequences

- This study provides pathological evidence of enteroviral infection among fatal myocarditis cases in a nationwide URTI cluster of cases associated with myocarditis and/or pericarditis

provide some evidence for an association between an enterovirus infection and sudden infant death, and furthermore suggest that molecular techniques may improve the diagnosis of myocarditis compared with the traditional histological criteria.

Fatal cases of myocarditis have been described previously in an enteroviral outbreak where myocarditis was not a major clinical manifestation. ${ }^{19}$ Our report is one of the few that describes pathological evidence of enteroviral infection among fatal myocarditis cases in a nationwide URTI cluster of cases associated with myocarditis and/or pericarditis. This cluster of acute myocarditis affected most of the Greek regions during a prolonged rainy season. It is interesting that fatal cases of myocarditis and/or pericarditis have not previously been reported in Greece in relation to seasonal increases of URTI.

Serology is usually not available in cases of sudden unexpected death as a result of viral myocarditis. It has been also stated that enterovirus is rarely cultured from the myocardium of postmortem tissues, and that the immunofluorescent detection of its antigen has seldom been successful. ${ }^{20} 21$ In our present study, histological and immunohistochemical results provided unequivocal evidence linking enteroviruses with the fatal cases of myopericarditis, and the IFA results confirmed in all three cases that coxsackievirus B3 was the causative agent. Although sequencing and comparison of the viral capsid region VPl was not carried out, nucleotide alignment of the $5^{\prime}$-UTR amplicons showed a $100 \%$ similarity to coxsackievirus B3 sequences. Adenovirus has been described as an important pathogen that could alone, or in dual infections with an enterovirus, be associated with fatalities during an enteroviral outbreak. ${ }^{22}{ }^{23}$ However, this viral agent (or others) was not detected as the cause of fatal myocarditis in our samples. Our data link coxsackievirus B3 infection and direct tissue damage in the three fatal myocarditis cases.

\section{ACKNOWLEDGEMENTS}

We thank Dr I Kourbeti for providing outbreak information data. We thank Mrs J Pournou and Mrs M Koutouvali for their expert technical assistance in the Department of Microbiology, Medical School, University of Athens, Greece.

The families of these patients gave their permission for the details to be published. 


\section{Authors' affiliations}

N Spanakis, A Tsakris, N J Legakis, Department of Microbiology, Medical School, University of Athens, 11527 Athens, Greece E N Manolis, Department of Anatomy and Histology, Faculty of Nursing, School of Health Sciences, University of Athens S Tsiodras, T Panagiotopoulos, G Saroglou, Hellenic Centre for Infectious Diseases Control, 10433 Athens, Greece

\section{REFERENCES}

1 Kandolf $\mathbf{R}$, Klingel K, Zell R, et al. Molecular mechanisms in the pathogenesis of enteroviral heart disease: acute and persistent infections. Clin Immunol Immunopathol 1993;68:153-8.

2 Martino TA, Liu P, Pertic M et al. Enteroviral myocarditis and dilated cardiomyopathy: a review of clinical and experimental studies. In: Rotbart HA, eds. Human enterovirus infections. Washington, DC: ASM Press, 1995:291-352

3 Grumbach IM, Heim A, Pring-Akerblom P, et al. Adenoviruses and enteroviruses as pathogens in myocarditis and dilated cardiomyopathy. Acta Cardiol 1999;54:83-8

4 Panagiotopoulos T, Tselentis Y, Gautrais A, et al. Cases of acute respiratory infection with myocarditis and pericarditis in Greece-April 2002. Eurosurveillance Weekly 26 April, 2002;6(17)

5 Panagiotopoulos T, Tsiodras S, Spala G, et al. Update-cases of acute respiratory infection with myocarditis and pericarditis in Greece. Eurosurveillance Weekly 3 May, 2002;6(18).

6 Avellon A, Perez P, Aguilar JC, et al. Rapid and sensitive diagnosis of human adenovirus infections by a generic polymerase chain reaction. J Virol Methods $2001 ; 92: 113-20$

7 Tenorio A, Echevarria JE, Casas I, et al. Detection and typing of human herpesviruses by multiplex polymerase chain reaction. J Virol Methods 1993:44:261-9.

8 Herrmann B, Larsson C, Zweygberg BW. Simultaneous detection and typing of influenza viruses $A$ and $B$ by a nested reverse transcription-PCR: comparison to virus isolation and antigen detection by immunofluorescence and optical immunoassay (FLU OIA). J Clin Microbiol 2001;39:134-8.

9 Read SJ, Kurtz JB. Laboratory diagnosis of common viral infections of the central nervous system by using a single multiplex PCR screening assay. J Clin Microbiol 1999;37:1352-5.
10 Aretz HT, Billingham ME, Edwards WD, et al. Myocarditis. A histopathological definition and classification. Am J Cardiovasc Pathol 1987;1:3-14.

11 Schoub BD, Johnson S, McAnerney JM, et al. Epidemic coxsackie B virus infection in Johannesburg, South Africa. J Hyg Lond 1985:95:447-55.

12 Li Y, Peng T, Yang Y, et al. High prevalence of enteroviral genomic sequence in myocardium from cases of endemic cardiomyopathy (Keshan disease) in China. Heart 2000;83:696-701

13 Schowengerdt KO, Ni J, Denfield SW, et al. Diagnosis, surveillance, and epidemiologic evaluation of viral infections in pediatric cardiac transplant recipients with the use of the polymerase chain reaction. J Heart Lung Transplant 1996;15:111-23.

14 Bowles NE, Ni J, Marcus F, et al. The detection of cardiotropic viruses in the myocardium of patients with arrhythmogenic right ventricular dysplasia/ cardiomyopathy. J Am Coll Cardiol 2002;39:892-5.

15 Andreoletti L, Bourlet T, Moukassa D, et al. Enteroviruses can persist with or without active viral replication in cardiac tissue of patients with end-stage ischemic or dilated cardiomyopathy. J Infect Dis 2000;182:1222-7.

16 Klein RM, Jiang $H_{1}$, Du M et al. Detection of enteroviral RNA (poliovirus types 1 and 3 ) in endomyocardial biopsies from patients with ventricular tachycardia and survivors of sudden cardiac death. Scand J Infect Dis 2002;34:746-52

17 Baasner A, Dettmeyer R, Graebe M, et al. PCR-based diagnosis of enterovirus and parvovirus $B 19$ in paraffin-embedded heart tissue of children with suspected sudden infant death syndrome. Lab Invest 2003;83:1451-5.

18 Dettmeyer R, Baasner A, Schlamann M, et al. Coxsackie B3 myocarditis in 4 cases of suspected sudden infant death syndrome: diagnosis by immunohistochemical and molecular-pathologic investigations. Pathol Res Pract 2002; 198:689-96.

19 Ho M, Chen ER, Hsu KH, et al. An epidemic of enterovirus 71 infection in Taiwan. N Engl J Med 1999;341:929-35.

20 Maze SS, Adolph RJ. Myocarditis: unresolved issues in diagnosis and treatment Clin Cardiol 1990:13:69-79.

21 Mounts AW, Amr S, Jamshidi R, et al. A cluster of fulminant myocarditis cases in children, Baltimore, Maryland, 1997. Pediatr Cardiol $2001 ; 22: 34-9$.

22 Cardosa MJ, Krishnan S, Tio PH, et al. Isolation of subgenus B adenovirus during a fatal outbreak of enterovirus 71-associated hand, foot, and mouth disease in Sibu, Sarawak. Lancet 1999;354:987-91.

23 Bowles NE, Ni J, Kearney DL, et al. Detection of viruses in myocardial tissues by polymerase chain reaction. Evidence of adenovirus as a common cause of myocarditis in children and adults. J Am Coll Cardiol 2003;42:466-72. 